\title{
Overexpression of COX6B1 protects against I/R-induced neuronal injury in rat hippocampal neurons
}

\author{
SHAN YANG ${ }^{1}$, PENG WU $^{2}$, JIANWEN XIAO $^{3}$ and LI JIANG ${ }^{2}$ \\ ${ }^{1}$ Department of Pediatrics, Nanchuan People's Hospital Affiliated to Chongqing Medical University, Chongqing 408400; \\ Departments of ${ }^{2}$ Neurology and ${ }^{3}$ Hematology, Children's Hospital Affiliated to Chongqing Medical University, \\ Chongqing 400014, P.R. China
}

Received June 15, 2018; Accepted March 27, 2019

DOI: $10.3892 / \mathrm{mmr} .2019 .10144$

\begin{abstract}
Cerebrovascular disease (CVD) is one of the leading causes of mortality worldwide. The role of cytochrome $\mathrm{c}$ oxidase subunit 6B1 (COX6B1) in the central nervous system remains unclear. The present study aimed to analyze the role of COX6B1 in rat hippocampal neurons extracted from fetal rats. The subcellular localization of the neuron-specific marker microtubule-associated protein 2 was detected by immunofluorescence assay. Cell viability was assessed using a cell counting kit, and the levels of apoptosis and cytosolic $\mathrm{Ca}^{2+}$ were analyzed by flow cytometry. The expression levels of the molecular factors downstream to COX6B1 were determined using reverse transcription-quantitative polymerase chain reaction and western blotting. Reoxygenation following oxygen-glucose deprivation (OGD) decreased cell viability and the expression levels of COX6B1 in a time-dependent manner, and 60 min of reoxygenation was identified as the optimal time period for establishing an ischemia/reperfusion (I/R) model. Overexpression of COX6B1 was demonstrated to reverse the viability of hippocampal neurons following $\mathrm{I} / \mathrm{R}$ treatment. Specifically, COX6B1 overexpression decreased the cytosolic concentration of $\mathrm{Ca}^{2+}$ and suppressed neuronal apoptosis, which were increased following I/R treatment. Furthermore, overexpression of COX6B1 increased the protein expression levels of apoptosis regulator BCL-2 and mitochondrial cytochrome c (cyt c), and decreased the protein expression levels of apoptosis regulator BCL2-associated $\mathrm{X}$ and cytosolic cyt $\mathrm{c}$ in I/R model cells. Collectively, the present study results suggested that COX6B1 overexpression may reverse I/R-induced neuronal damage by increasing the viability of neurons, by decreasing the cytosolic levels of $\mathrm{Ca}^{2+}$ and by suppressing apoptosis. These results may facilitate
\end{abstract}

Correspondence to: $\mathrm{Dr} \mathrm{Li}$ Jiang, Department of Neurology, Children's Hospital Affiliated to Chongqing Medical University, 136 Zhongshan Second Road, Chongqing 400014, P.R. China E-mail: lijiang_jll@163.com

Key words: cerebrovascular disease, cytochrome c oxidase subunit 6B1, hippocampal neurons, apoptosis the development of novel strategies for the prevention and treatment of CVD.

\section{Introduction}

Cerebrovascular disease (CVD) is a common disease affecting the nervous system and may be caused by various types of cerebrovascular injuries (1). Notably, CVD morbidity increased over the past years $(2,3)$. In addition, CVD may affect the quality of life, and $\sim 40 \%$ of patients with CVD present severe disabilities (4). Ischemic cerebrovascular disease accounts for $\sim 75 \%$ of all CVD cases and presents high disability, mortality and recurrence rates (5).

Treatments aimed to re-establish blood flow and blood perfusion in the ischemic penumbra following cerebral ischemia are necessary to improve the outcomes of patients with CVD (6,7). Previous studies demonstrated that metabolic disorders, oxidative stress, neurotoxicity of the excitatory amino acids, inflammatory cell infiltration, cell apoptosis, microvascular neovascularization and additional factors are involved in the development of cerebral ischemia-reperfusion (I/R) injury $(8,9)$. The structure and the function of the vasculature may be severely impaired following I/R injury, influencing the permeability of the blood-brain barrier and the mechanisms underlying vascular homeostasis; these processes may aggravate cerebral edema, leading to a decline in the clinical outcomes of the patients $(6,10,11)$. Therefore, reducing reperfusion injury following cerebral infarction is an important topic of research.

Cytochrome c oxidase subunit 6B1 (COX6B1) is one of the subunits of the cytochrome c oxidase (COX) and is expressed in numerous cell types, including yeast and HeLa cells (12). The human COX6B1 gene is located on chromosome 19q13; the coding sequence comprises 261 base pairs and the protein contains 87 amino acids (12-15). The principal role of COX6B1 is to connect two COX monomers to form a dimer, serving a role in the cell respiratory chain (16). Alterations in the dimeric structure of the COX complex affects its function, and defects in the assembly of a functional dimer may cause severe diseases $(17,18)$. A previous study demonstrated that genetic mutations in the conserved regions of COX6B1 may lead to mitochondrial encephalopathy (16). Mitochondrial diseases are frequently associated with mutations in or an absence of 
COX (19). Additionally, previous studies reported that abnormal alternations of COX6B1 markedly affect the function of COX, potentially leading to the occurrence of cerebromyopathy, hydrocephalus and other diseases $(16,17)$. COX6B1 protein may be associated with the development of the central nervous system (16). Previous studies reported that COX6B1 may protect the myocardium against $\mathrm{I} / \mathrm{R}$ injury by regulating mitochondrial function $(20,21)$; however, whether COX6B1 protects hippocampal neurons against I/R injury has not been reported, and the molecular mechanisms underlying the role of COX6B1 in the development of the nervous system remains unclear.

In the present study, a model of I/R injury was constructed using rat hippocampal neurons. Additionally, the molecular mechanisms underlying the role of COX6B1 following I/R injury in hippocampal neurons was investigated.

\section{Materials and methods}

Animals. A total of 6 pregnant Sprague-Dawley (SD) rats at gestational day 18 (age, 2 months; weight, 180-200 g) were obtained from Guangdong Medical Laboratory Animal Center (Foshan, China) and maintained at $22 \pm 1^{\circ} \mathrm{C}, 40-70 \%$ humidity, with free access to food and water, under a 12-h light/dark cycle. The animal experiments were approved by The Ethics Committee of The Nanchuan People's Hospital Affiliated to Chongqing Medical University (Chongqing, China).

Extraction of hippocampal neurons. Pregnant SD rats were anesthetized by ether, and 12 fetal rats were extracted. The fetal rats were transferred to Petri dishes containing $75 \%$ ethanol and the heads were removed using a guillotine (cat. no. 7950; Ugo Basile SRL, Gemonio, Italy). Subsequently, the hippocampi were dissected and digested with $2.5 \mathrm{~g} / 1$ trypsin (Beyotime Institute of Biotechnology, Haimen, China) for $15 \mathrm{~min}$ at $37^{\circ} \mathrm{C}$. Dulbecco's modified Eagle's medium/Ham's F-12 nutrient mixture (DMEM/F12; Gibco; Thermo Fisher Scientific, Inc., Waltham, MA, USA) supplemented with $10 \%$ fetal bovine serum (FBS; Gibco; Thermo Fisher Scientific, Inc.) was added to the tissues and gently agitated. Cell suspension was filtered through a 400- $\mu \mathrm{m}$ nylon mesh sieve, centrifuged at $1,000 \mathrm{x} \mathrm{g}$ for $5 \mathrm{~min}$ and resuspended in DMEM/F12 at a density of $1 \times 10^{6}$ cells $/ \mathrm{ml}$. The neurons obtained were cultured in an incubator under a humidified atmosphere containing $5 \% \mathrm{CO}_{2}$ at $37^{\circ} \mathrm{C}$ for $24 \mathrm{~h}$. Following a $24-\mathrm{h}$ incubation, DMEM/F12 was replaced with neurobasal medium containing $2 \% \mathrm{~B}-27$ supplement (Thermo Fisher Scientific, Inc.) and $5 \mu \mathrm{mol} / \mathrm{l}$ cytosine arabinoside (Sigma-Aldrich; Merck KGaA, Darmstadt, Germany) was added to inhibit glial cell proliferation; cells were subsequently incubated for $48 \mathrm{~h}$ at $37^{\circ} \mathrm{C}$. Following a $48-\mathrm{h}$ incubation, the medium was replaced, and cells were cultured until further experimentation; $50 \%$ of the culture medium was replaced every 3 days.

Immunofluorescence assay. Following culturing, hippocampal neurons were fixed with $4 \%$ paraformaldehyde at room temperature for $20 \mathrm{~min}$ on glass coverslips. PBS containing $0.2 \%$ Triton X-100 (Thermo Fisher Scientific, Inc.) was used for permeabilizing cells for $10 \mathrm{~min}$. Cells were blocked with $5 \%$ bovine serum albumin (Bovogen Biologicals Pty Ltd., Victoria, Australia) at room temperature for $25 \mathrm{~min}$. The cells were incubated with anti-microtubule-associated protein 2 (MAP2) antibody (1:100; cat. no. ab32454; Abcam, Cambridge, UK) at $4^{\circ} \mathrm{C}$ for $24 \mathrm{~h}$, and washed with PBS three times. Subsequently, the cells were incubated with goat anti-rabbit fluorescein isothiocyanate (FITC)-conjugated immunoglobulin G (IgG; 1:5,000; cat. no. ab6717; Abcam) at $37^{\circ} \mathrm{C}$ for $1 \mathrm{~h}$. Following incubation, the cells were washed with PBS three times, and subsequently incubated with DAPI (Thermo Fisher Scientific, Inc.) at room temperature for $10 \mathrm{~min}$. Stained samples were observed under an MF43 fluorescence microscope (magnification, x200 and x400; Micro-shot Technology Limited, Guangzhou, China).

Oxygen-glucose deprivation/reoxygenation $(O G D / R)$ in vitro model establishment. Hippocampal neurons $\left(1 \times 10^{6}\right.$ cells $\left./ \mathrm{ml}\right)$ were cultured in neurobasal medium containing $2 \%$ B-27 supplement at $37^{\circ} \mathrm{C}$ with $5 \% \mathrm{CO}_{2}$ for 10 days and cells were exposed to OGD by replacing the neurobasal medium with Earle's balanced salt solution (EBSS) without glucose. The cells were maintained in an anaerobic chamber (YQX-II; Shanghai CIMO Medical Instrument Co., Ltd., Shanghai, China) with $95 \% \mathrm{~N}_{2}$ and $5 \% \mathrm{CO}_{2}$ for $60 \mathrm{~min}$ at $37^{\circ} \mathrm{C}$. Subsequently, cells were subjected to reoxygenation treatment (incubation in neurobasal medium in an incubator with $5 \% \mathrm{CO}_{2}$ at $37^{\circ} \mathrm{C}$ ) for 10,30 or $60 \mathrm{~min}$ prior to further experimentation.

Cell viability analysis. Following the aforementioned OGD/R treatment, a Cell Counting Kit-8 (CCK-8) was performed to determine the viability of cells following OGD and OGD/R treatment. The hippocampal neurons $\left(2 \times 10^{3}\right.$ cells/well $)$ were seeded into 96-well plates and maintained in an incubator for $24 \mathrm{~h}$ at $37^{\circ} \mathrm{C}$. Following incubation, $10 \mu \mathrm{l} \mathrm{CCK}-8$ reagent (Beijing Solarbio Science \& Technology Co., Ltd., Beijing, China) was added into each well. Cells were transferred into the incubator and maintained for $2 \mathrm{~h}$ at $37^{\circ} \mathrm{C}$. A microplate reader (Bio-Rad Laboratories, Inc., Hercules, CA, USA) was used to detect the absorbance at $450 \mathrm{~nm}$.

Cell transfection. The pcDNA3.1(+)-empty vector (EV) and pcDNA3.1(+)-COX6B1 vector were purchased from GenomeDitech Co., Ltd., (Shanghai, China). Cells were seeded ( $2 \times 10^{5}$ cells/well) into 6 -well plates, serum-starved overnight, and subsequently transfected with COX6B1 vector $(20 \mathrm{nM})$ using Lipofectamine ${ }^{\circledR} 3000$ (Invitrogen; Thermo Fisher Scientific, Inc.). Cells were transfected for $36 \mathrm{~h}$ at $37^{\circ} \mathrm{C}$ prior to subsequent experimentation. Cells were transfected with the plasmids prior to OGD/R.

Cell grouping. To assess the effects of OGD/R treatment on neurons, cells were divided into five experimental groups: i) Control group, in which untreated neurons were exposed to PBS; ii) OGD group, in which cells were exposed to OGD for $60 \mathrm{~min}$, as aforementioned, without reoxygenation; iii) OGD-10 group, OGD cells exposed to reoxygenation treatment for $10 \mathrm{~min}$; iv) OGD-30 group, OGD cells exposed to reoxygenation treatment for $30 \mathrm{~min}$; v) OGD-60 group, OGD cells exposed to reoxygenation treatment for $60 \mathrm{~min}$. Based upon the results of this experiment, OGD-60 treatment was selected to be the I/R injury model in subsequent experiments. To investigate the role of COX6B1 in OGD/R-induced damage, cells were divided into six groups: i) Control 
group, untreated cells; ii) EV group, cells transfected with pcDNA3.1(+)-EV; iii) COX6B1 group, cells transfected with pcDNA3.1(+)-COX6B1 overexpression vector; iv) $\mathrm{EV}+\mathrm{I} / \mathrm{R}$ group, OGD-60 cells transfected with pcDNA3.1(+)-EV; v) I/R group, untransfected OGD-60 cells; and vi) COX6B1 + I/R group, OGD-60 cells transfected with pcDNA3.1(+)-COX6B1. All experiments were performed at least three times.

Cytosolic $\mathrm{Ca}^{2+}$ levels analysis. The cytosolic $\mathrm{Ca}^{2+}$ levels of cells in the control, EV, COX6B1, I/R, EV + I/R and COX6B1 + I/R groups were determined by flow cytometry. Following transfection, hippocampal neurons were seeded $\left(2 \times 10^{4}\right.$ cells/well $)$ into 6-well plates and cultured in an incubator for $24 \mathrm{~h} 37^{\circ} \mathrm{C}$ prior to exposure to the aforementioned OGD/R protocol. The staining reagent Fluo-3, AM (Sigma-Aldrich; Merck KGaA) was added into the wells and the cells were incubated at $37^{\circ} \mathrm{C}$ for $45 \mathrm{~min}$. Subsequently, the cells were incubated with Hank's Balanced Salt Solution (HBSS; Thermo Fisher Scientific, Inc.) containing 10\% FBS (Gibco; Thermo Fisher Scientific, Inc.) for $40 \mathrm{~min}$ at $37^{\circ} \mathrm{C}$. Following incubation, the cells were washed with HBSS for 3 times. The cells were resuspended in HBSS and incubated at $37^{\circ} \mathrm{C}$ for $10 \mathrm{~min}$. The concentration of intracellular $\mathrm{Ca}^{2+}$ was assessed by flow cytometry (BD Biosciences, San Jose, CA, USA) and FSC Express version 3 software (De Novo Software, Glendale, CA, USA).

Cell apoptosis analysis. The cell apoptosis of cells in the control, EV, COX6B1, I/R, EV + I/R and COX6B1 + I/R groups were determined by flow cytometry. Following transfection, hippocampal neurons were seeded $\left(2 \times 10^{4}\right.$ cells/well) into 6-well plates and cultured in an incubator for $24 \mathrm{~h}$ prior to exposure to the aforementioned OGD/R protocol. The cells were then incubated with Annexin V-FITC and propidium iodide (Beijing Solarbio Science \& Technology Co., Ltd.) in the dark for $20 \mathrm{~min}$ at room temperature. A flow cytometer and FSC Express version 3 software were used to measure the level of apoptosis. Advanced apoptotic cells were presented in upper right quadrants, and early apoptotic cells in lower right quadrants. The relative apoptosis rate of total apoptotic cells (early and advanced) was calculated.

Western blotting. A Mitochondria/Cytosol Fractionation kit (AmyJet Scientific Inc., Wuhan, China) was used to separate the mitochondrial and cytosolic fractions. Total protein was extracted from neurons $\left(2 \times 10^{4}\right.$ cells/well in 6 -well plates $)$ using radioimmunoprecipitation assay lysis buffer (Beyotime Institute of Biotechnology). The protein concentration was determined using Bradford method (Beyotime Institute of Biotechnology). A total of $30 \mu \mathrm{g}$ protein from each sample was separated by $12 \%$ SDS-PAGE and transferred to a polyvinylidene fluoride (PVDF) membrane (EMD Millipore, Billerica, MA, USA). The PVDF membrane was blocked using $5 \%$ skimmed milk at $37^{\circ} \mathrm{C}$ for $60 \mathrm{~min}$. Subsequently, the membranes were incubated at $4^{\circ} \mathrm{C}$ for $24 \mathrm{~h}$ with the following primary antibodies: Anti-COX6B1 (1:600; cat. no. 11425-1-AP; ProteinTech Group, Inc., Chicago, IL, USA), apoptosis regulator BCL-2 (1:1,000; cat. no. ab194583; Abcam), anti-BCL2-associated X, apoptosis regulator (BAX; 1:800; cat. no. ab53154; Abcam), anti-cytochrome c (cyt c; 1:600; cat. no. ab13575; Abcam), anti-cytochrome c oxidase
Table I. Primer sequences used in reverse transcription-quantitative polymerase chain reactions.

\begin{tabular}{|c|c|c|}
\hline Gene & Primer sequence $\left(5^{\prime} \rightarrow 3^{\prime}\right)$ & $\begin{array}{c}\text { Product } \\
\text { size (bp) }\end{array}$ \\
\hline \multirow{2}{*}{ COX6B1 } & F: AAGAACTACAAAACCGCCCC & 195 \\
\hline & R: ATCCCAGGCTGAGACCCAT & \\
\hline \multirow[t]{2}{*}{ BAX } & F: GAGACACCTGAGCTGACCTT & 187 \\
\hline & R: CGTCTGCAAACATGTCAGCT & \\
\hline \multirow[t]{2}{*}{ BCL-2 } & F: GCCTTCTTTGAGTTCGGTGG & 221 \\
\hline & R: CTGAGCAGCGTCTTCAGAG & \\
\hline \multirow[t]{2}{*}{ Cyt c } & F: GGAGGCAAGCATAAGACTGG & 210 \\
\hline & R: TGCCCTTTCTCССТTCTTCT & \\
\hline \multirow[t]{2}{*}{$\beta$-actin } & F: TGTGTTGTCCCTGTATGCC & 232 \\
\hline & R: AATGTCACGCACGATTTCCC & \\
\hline
\end{tabular}

BAX, BCL2-associated X, apoptosis regulator; BCL-2, apoptosis regulator $\mathrm{BCL} 2$; bp, base pairs; COX6B1, cytochrome c oxidase subunit 6B1; cyt c, cytochrome c; F, forward; R, reverse.

subunit 4I1 (COX4I1; 1:1,000; cat. no. ab33985; Abcam) and anti- $\beta$-actin (1:800; cat. no. ab8226; Abcam). Subsequently, the PVDF membranes were incubated at room temperature for $1.5 \mathrm{~h}$ with one of the following secondary antibodies: Rabbit anti-mouse IgG (1:7,000; cat. no. 58802; Cell Signaling Technology, Inc., Danvers, MA, USA) or goat anti-rabbit IgG (1:700; cat. no. ab6721; Abcam). Protein bands were visualized with Enhanced Chemiluminescent reagents (EMD Millipore) and the protein expression level was detected using a Molecular Imager ${ }^{\circledR}$ Gel Doc ${ }^{\mathrm{TM}}$ XR+ System (cat. no. 1708195; Bio-Rad Laboratories, Inc.) and ImageJ version 1.46 software (National Institutes of Health, Bethesda, MD, USA). $\beta$-actin was used as the loading control for total proteins and cytosolic fractions, whereas COX4I1 was used as the loading control for the mitochondrial fraction; protein expression was normalized to $\beta$-actin and COX4I1.

Reverse transcription-quantitative polymerase chain reaction $(R T-q P C R)$. A Mitochondria/Cytosol Fractionation kit was used to separate the mitochondrial and cytosolic fractions. Total RNA was extracted from hippocampal neurons $\left(2 \times 10^{4}\right.$ cells/well in 6 -well plates) using TRIzol ${ }^{\circledR}$ reagent (Thermo Fisher Scientific,Inc.). RNA was reverse transcribed to cDNA using TIANScript cDNA Synthesis kit (Tiangen Biotech Co., Ltd., Beijing, China) according to the manufacturer's protocol. cDNA was amplified using TeloPrime Full-Length cDNA amplification kit (Lexogen GmbH, Vienna, Austria), according to the manufacturer's protocol. qPCR experiments were performed using a SYBR Premix Ex Taq ${ }^{\mathrm{TM}}$ Real-Time PCR kit (Takara Bio, Inc., Otsu, Japan). qPCR thermocycling conditions were as follows: Initial denaturation at $95^{\circ} \mathrm{C}$ for $1 \mathrm{~min}$, followed by 45 cycles at $96^{\circ} \mathrm{C}$ for $15 \mathrm{sec}$ and at $63^{\circ} \mathrm{C}$ for $45 \mathrm{sec}$, with a final extension step at $75^{\circ} \mathrm{C}$ for $10 \mathrm{~min}$. $\beta$-actin was used as the reference gene, except for mitochondrial fraction, for which COX4I1 was used as the reference gene. The primers were purchased from Sigma-Aldrich (Merck KGaA) and are listed in Table I. The relative expression of genes was 


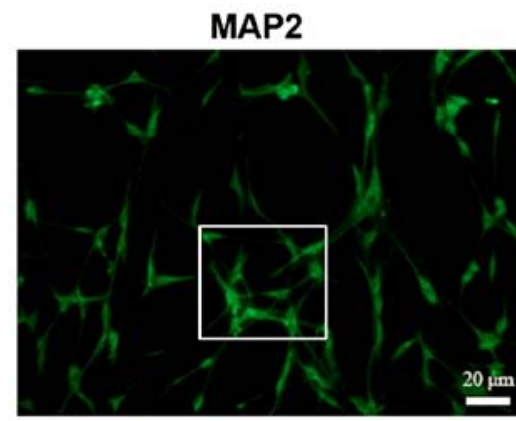

MAP2

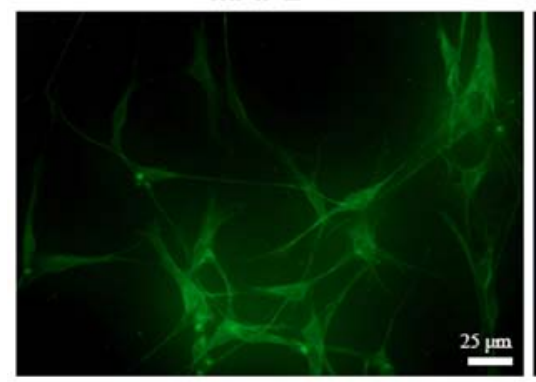

DAPI

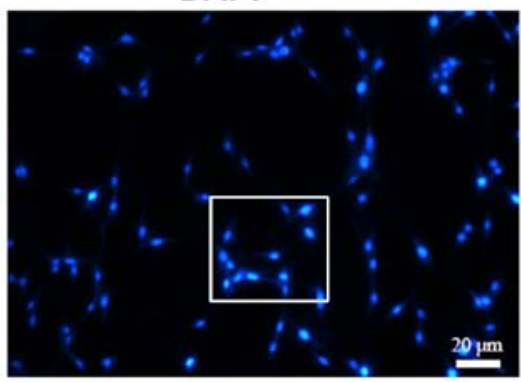

DAPI

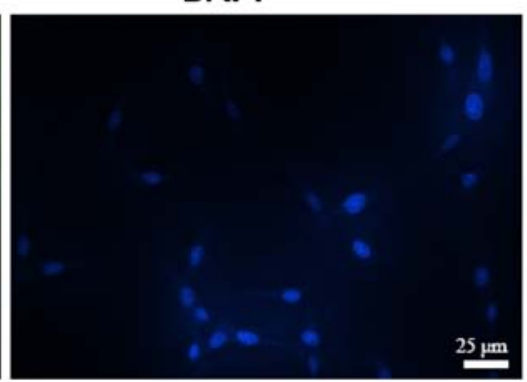

Merge

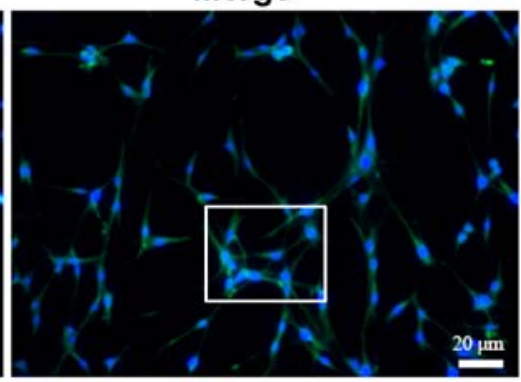

Merge

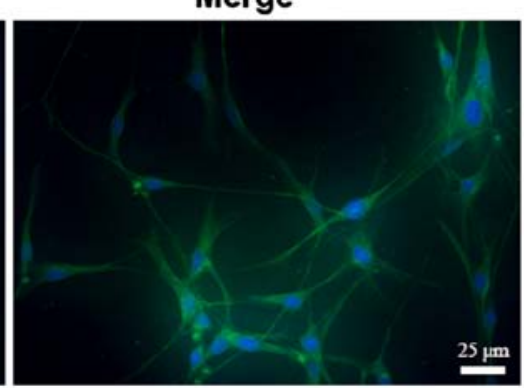

Figure 1. Identification of hippocampal neurons. Immunofluorescence assay was performed to verify the protein expression of MAP2, a marker of hippocampal neurons; DAPI was used to stain the nuclei. MAP2, microtubule associated protein 2.

calculated using the $2^{-\Delta \Delta \mathrm{Cq}}$ quantification method (22) and normalized to $\beta$-actin and COX4I1 expression.

Statistical analysis. All experiments were performed at least three times. GraphPad Prism 6.0 (GraphPad Software, Inc., La Jolla, CA, USA) was used to perform statistical analysis. Data are presented as the mean \pm standard deviation. Statistical comparisons were performed using one-way analysis of variance followed by Tukey's test. $\mathrm{P}<0.05$ was considered to indicate a statistically significant difference.

\section{Results}

Cell viability and COX6B1 expression levels are decreased in hippocampal neurons exposed to $O G D / R$. The cellular identity of the extracted hippocampal neurons was tested by immunofluorescence assay and $>95 \%$ of cells were identified to be positive for MAP2 (Fig. 1). The effects of OGD and OGD/R on hippocampal neurons were investigated, and cell viability and COX6B1 mRNA and protein expression levels were examined by CCK-8 assay, RT-qPCR analysis and western blotting, respectively. Following exposure to OGD, cell viability decreased significantly in hippocampal neurons compared with the Control group (Fig. 2A). Furthermore, the viability of cells in the OGD-10 and OGD-30 groups was markedly decreased compared with in OGD group, and significantly decreased in the OGD-60 group. The mRNA and protein expression levels of COX6B1 were significantly reduced in the OGD group, compared with the Control group (Fig. 2B); additionally, the mRNA and protein expression levels of COX6B1 were significantly in OGD-30 and -60 groups compared with in the OGD group (Fig. 2C and D). As OGD-60 treatment exhibited the most pronounced effects on cell viability and COX6B1 expression, it was selected as the $\mathrm{I} / \mathrm{R}$ injury model in subsequent experiments.
Overexpression of $C O X 6 B 1$ decreases the cytosolic levels of $\mathrm{Ca}^{2+}$ in hippocampal neurons increased following $I / R$. The transfection efficiency of COX6B1 overexpression vectors was detected using RT-qPCR and western blotting. Hippocampal neurons transfected with pcDNA3.1(+)-COX6B1 exhibited an increase in the mRNA and protein expression levels of COX6B1 compared with the control EV-transfected cells (Fig. 3). To investigate the role of COX6B1 in the intracellular levels of $\mathrm{Ca}^{2+}$ in hippocampal neurons, flow cytometry was conducted. The cytosolic levels of $\mathrm{Ca}^{2+}$ were $4.19 \%$ in the Control group, 4.23 in $\mathrm{EV}, 3.67$ in COX6B1, 8.44 in $\mathrm{I} / \mathrm{R}, 8.15$ in $\mathrm{EV}+\mathrm{I} / \mathrm{R}$ and $6.02 \%$ in COX6B1 $+\mathrm{I} / \mathrm{R}$ treated cells (Fig. 4A). The relative concentration of $\mathrm{Ca}^{2+}$ was significantly increased in the I/R group compared with the EV group (Fig. 4B); however, COX6B1 overexpression decreased the concentration of $\mathrm{Ca}^{2+}$ compared with the $\mathrm{EV}+\mathrm{I} / \mathrm{R}$ group.

Overexpression of $C O X 6 B 1$ represses $I / R$-induced apoptosis of hippocampal neurons and increases cell viability. To investigate the effects of COX6B1 on apoptosis and viability of hippocampal neurons, flow cytometry and CCK-8 assays were conducted, respectively. Cells exposed to I/R exhibited an increased total apoptotic rate (early + advanced) compared with the EV group (Fig. 5A and B). Nevertheless, COX6B1 overexpression in hippocampal neurons decreased the apoptosis induced by I/R injury (Fig. 5A and B). In addition, cell viability was decreased following I/R injury, whereas overexpression of COX6B1 increased the viability of cells following I/R injury (Fig. 5C).

Overexpression of COX6B1 increases the expression levels of BCL-2 and decreases the expression levels of $B A X$ in hippocampal neurons following $I / R$ injury. To investigate apoptotic pathway components downstream of COX6B1 in hippocampal neurons, the expression levels 


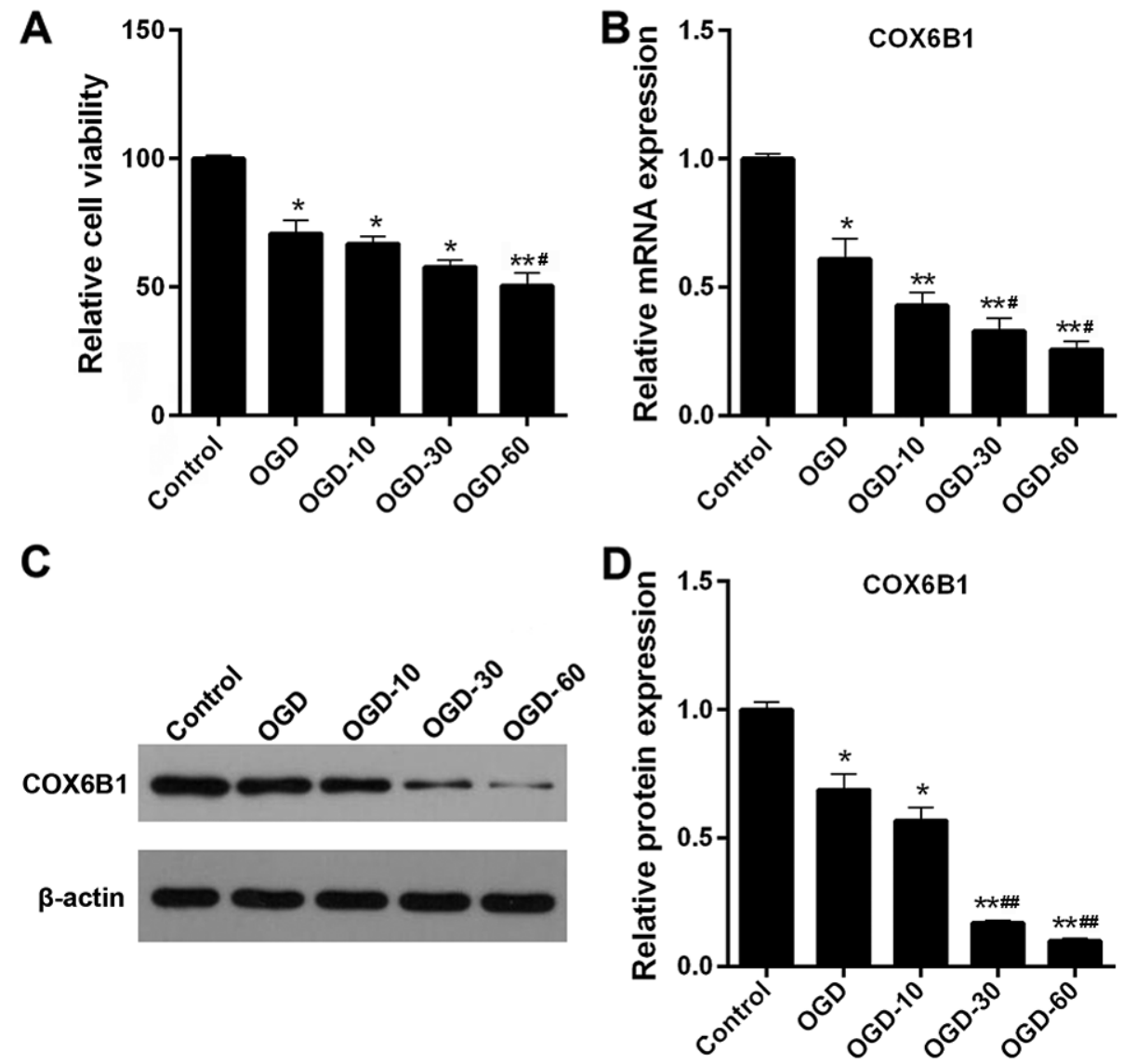

Figure 2. Cell viability and COX6B1 expression levels are decreased in hippocampal neurons exposed to OGD and reoxygenation. Hippocampal neurons were exposed to OGD with or without subsequent reoxygenation for various lengths of time. (A) Cell viability was assessed by Cell Counting Kit-8 assay. (B) mRNA expression level of COX6B1 was analyzed by reverse transcription-quantitative polymerase chain reaction. Protein expression level of COX6B1 was assessed using (C) western blotting and (D) densitometric analysis. ${ }^{\mathrm{P}}<0.05$ and ${ }^{* *} \mathrm{P}<0.01$ vs. Control; ${ }^{*} \mathrm{P}<0.05$ and ${ }^{\# \#} \mathrm{P}<0.01$ vs. OGD. COX6B1, cytochrome c oxidase subunit 6B1; OGD, oxygen-glucose deprivation; OGD-10/30/60, OGD cells receiving reoxygenation treatment for 10, 30 or 60 min, respectively.

A

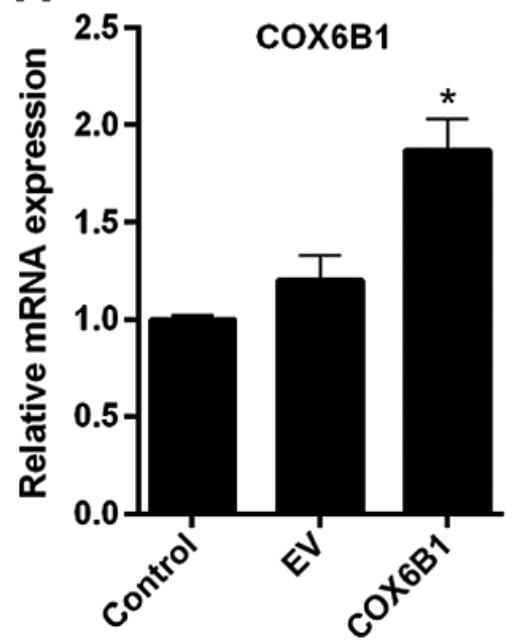

B

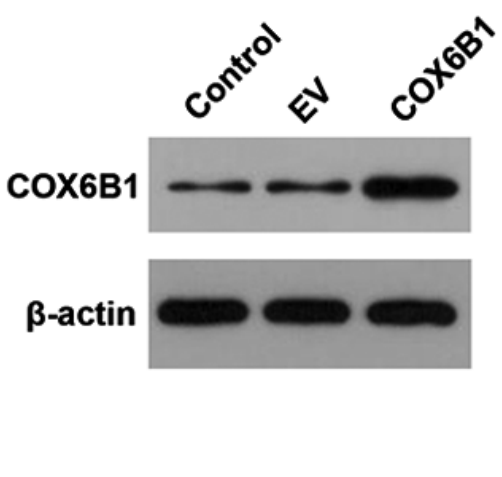

C

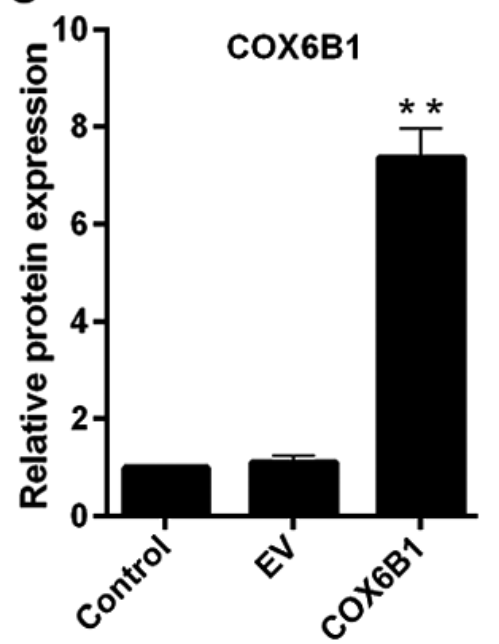

Figure 3. Transfection efficiency of COX6B1 in hippocampal neurons. Cells were transfected with pcDNA3.1(+)-EV and pcDNA3.1(+)-COX6B1. (A) COX6B1 mRNA expression levels were determined using reverse transcription-quantitative polymerase chain reaction. (B and C) COX6B1 protein expression levels were assessed using (B) western blotting and (C) densitometric analysis. $\mathrm{n}=4$; ${ }^{\mathrm{P}} \mathrm{P}<0.05$ and ${ }^{* *} \mathrm{P}<0.01$ vs. EV. COX6B1, cytochrome c oxidase subunit $6 \mathrm{~B} 1$; EV, empty vector.

of apoptosis-associated factors BCL-2 and BAX were investigated by RT-qPCR and western blotting. The mRNA expression levels of BAX in the I/R, EV + I/R and COX6B1 +
I/R groups were significantly increased compared with those in the EV group, whereas the expression levels of BCL-2 were significantly decreased compared with in the EV group 

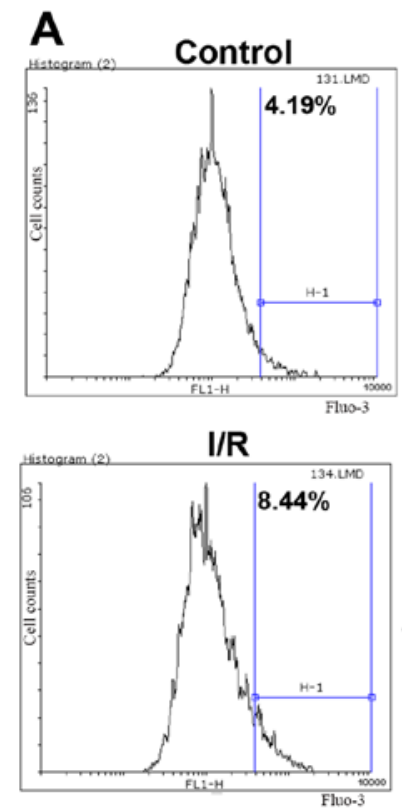

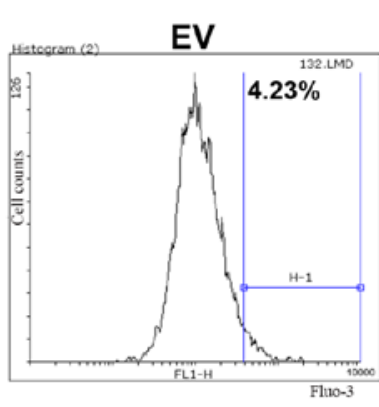

$E V+I / R$

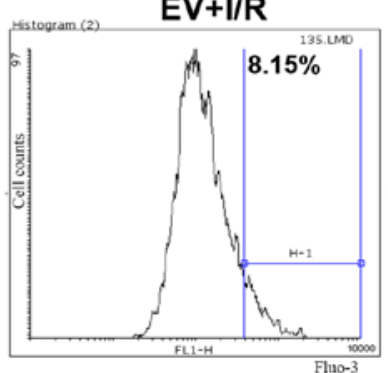

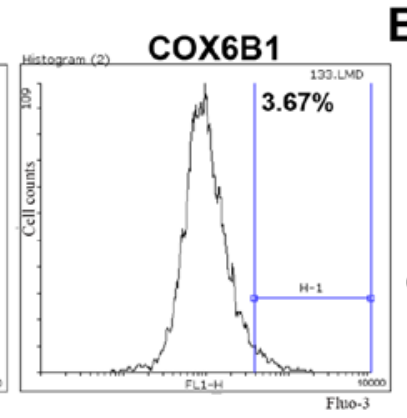

COX6B1+l/R

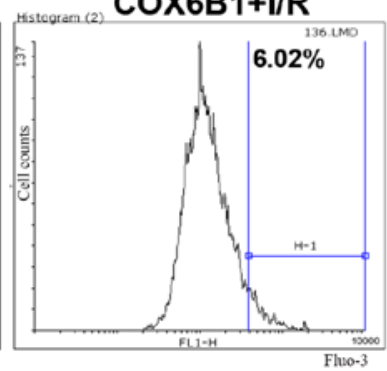

B

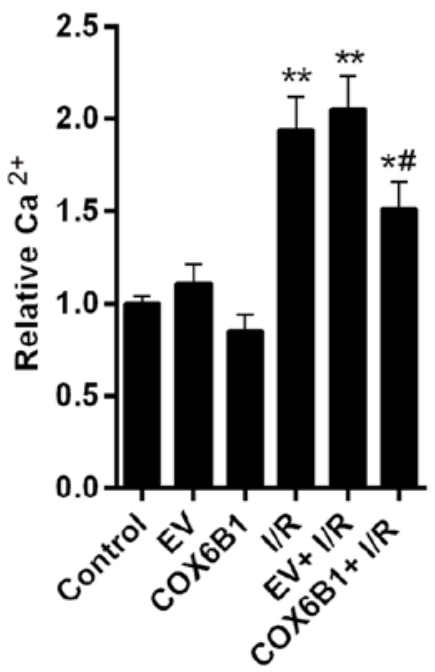

Figure 4. Overexpression of COX6B1 decreases the level of $\mathrm{Ca}^{2+}$ in hippocampal neurons following I/R injury. (A) Cells were exposed to OGD for 60 min, reoxygenated for $60 \mathrm{~min}$ and transfected with pcDNA3.1(+)-EV or with pcDNA3.1(+)-COX6B1 overexpression plasmid. Ca ${ }^{2+}$ concentration levels in hippocampal neurons were measured using flow cytometry. (B) Relative $\mathrm{Ca}^{2+}$ concentrations for each group from $\mathrm{Part}(\mathrm{A}) . \mathrm{n}=4$; ${ }^{*} \mathrm{P}<0.05$ and ${ }^{* *} \mathrm{P}<0.01$ vs. $\mathrm{EV}$; ${ }^{\#} \mathrm{P}<0.05$ vs. EV + I/R. COX6B1, cytochrome c oxidase subunit $6 \mathrm{~B} 1 ; \mathrm{EV}$, empty vector; I/R, ischemia/reperfusion.

(Fig. 6A and B, respectively). Overexpression of COX6B1 following I/R injury decreased the mRNA expression levels of BAX and increased the expression levels of BCL-2 compared with the $\mathrm{EV}+\mathrm{I} / \mathrm{R}$ group. Similar alterations in the expression of BAX and BCL-2 were observed at the protein level, as assessed by western blotting (Fig. 6C-E).

Overexpression of $C O X 6 B 1$ increases the expression levels of mitochondrial cyt $c$ and decreases the expression levels of cytosolic cyt $c$ in hippocampal neurons following $I / R$. To investigate the role of COX6B1 on the expression levels of cyt $\mathrm{c}$ in hippocampal neurons, RT-qPCR and western blotting were performed. The expression levels of mitochondrial cyt c decreased, whereas the expression levels of cytosolic cyt c increased following I/R injury; COX6B1 overexpression reversed the effects of I/R injury (Fig. 7).

\section{Discussion}

Primary cultured hippocampal neurons are frequently harvested from hippocampi of newborn ( $<24$-h-old) rodents, owing to their localization and the amount of available material $(23,24)$, and these cells have been used to establish experimental models to study the function of neurons in vitro. Therefore, in the present study, the hippocampal neurons from fetal rats were selected as a model to study I/R injury. MAP2 is a specific marker of hippocampal neurons (25). MAP2 protein expression was used to confirm the identity of the extracted cells used in the present study; therefore, the extracted cells were identified as hippocampal neurons. OGD exposure has been used by various previous studies to investigate neuronal ischemia in vitro $(26,27)$. Therefore, in the present study, this method was selected to establish an I/R model in hippocampal neurons. Cell viability decreased by $\sim 50 \%$ in cells exposed to OGD for 60 min followed by reoxygenation treatment for $60 \mathrm{~min}$, as assessed by CCK- 8 assay.

Massa et al (16) demonstrated that genetic mutations affecting the 20th amino acid residue of COX6B1 protein may cause severe infantile encephalomyopathies. In addition, previous studies reported that knockout of COX5A and COX6A may lead to a decline in the number of neurons in animal models $(28,29)$. Nevertheless, the molecular mechanism underlying COX6B1 function in hippocampal neurons remains unclear. In the present study, the expression levels of COX6B1 decreased following ischemic or I/R injury. Therefore, it was hypothesized that COX6B1 may serve a role in hippocampal neurons following I/R injury. Subsequently, in the present study, COX6B1 was overexpressed in hippocampal neurons exposed to OGD/R, which reversed the I/R-induced decrease in viability of hippocampal neurons.

Dysregulation of intracellular $\mathrm{Ca}^{2+}$ homeostasis is a mechanism of cell death caused by various factors $(30,31)$. Cerebral ischemia leads to membrane depolarization and causes the release of presynaptic excitatory transmitters, including glutamic acid and aspartic acid, and extracellular $\mathrm{Ca}^{2+}$ ions enter the cells via voltage-gated channels and N-methyl-D-aspartate receptor-gated channels (32). An increase in intracellular $\mathrm{Ca}^{2+}$ may lead to the activation of $\mathrm{Ca}^{2+}$-dependent enzymes, including cytoplasmic phospholipases and proteases, which may cause the degradation of cell membranes and the disruption of the neuronal cytoskeleton, damaging the structure and function of neurons $(33,34)$. In the present study, the levels of cytosolic $\mathrm{Ca}^{2+}$ were increased following I/R neuronal injury, whereas overexpression of COX6B1 significantly decreased I/R-induced cytosolic $\mathrm{Ca}^{2+}$ levels in hippocampal neurons. The present results suggested that COX6B1 overexpression protected against I/R-induced neuronal damage by inhibiting the cytosolic levels of $\mathrm{Ca}^{2+}$. 

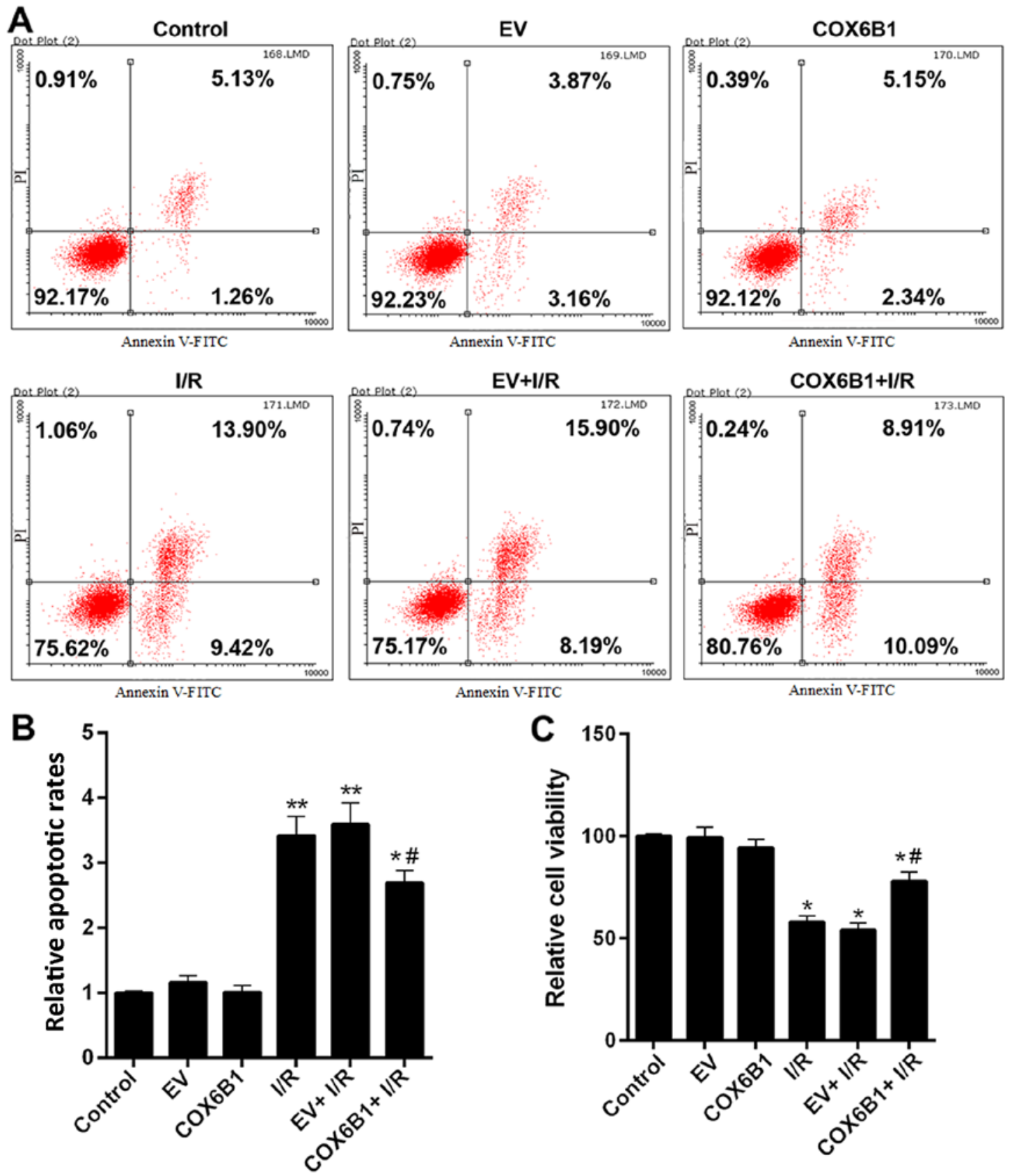

Figure 5. Overexpression of COX6B1 represses the apoptosis of hippocampal neurons induced by I/R injury and increased cell viability. (A-C) Cells were exposed to OGD for $60 \mathrm{~min}$, reoxygenated for $60 \mathrm{~min}$ and transfected with pcDNA3.1(+)-EV or with pcDNA3.1(+)-COX6B1 overexpression plasmid. (A) Cell apoptosis was assessed by flow cytometry. (B) Quantification of apoptosis. (C) Cell viability was detected by Cell Counting Kit- 8 assay. $\mathrm{n}=4$; " $\mathrm{P}<0.05$ and ${ }^{* *} \mathrm{P}<0.01$ vs. EV; ${ }^{*} \mathrm{P}<0.05$ vs. EV + I/R. COX6B1, cytochrome c oxidase subunit $6 \mathrm{~B} 1 ; \mathrm{EV}$, empty vector; FITC, fluorescein isothiocyanate; I/R, ischemia/reperfusion; PI, propidium iodide.

A number of previous studies demonstrated that neuronal apoptosis served a principal role in neuronal death following cerebral I/R $(26,35,36)$. In accordance with these previous studies, the present study demonstrated that apoptosis was promoted following I/R injury. Additionally, overexpression of COX6B1 was able to suppress I/R-induced neuronal apoptosis. Subsequently, the molecular mechanism underlying neuronal apoptosis was investigated in the present study. The proteins belonging to the BCL-2 family are involved in the apoptotic pathway, and regulate the permeability of the outer membranes of mitochondria (37). BCL-2 and BAX are antagonistic proteins. BCL-2 serves anti-apoptotic roles, whereas BAX serves a role in promoting apoptosis (38). Aboutaleb et al (39) demonstrated that the expression level of BCL-2 decreased, whereas BAX expression increased in hippocampal neurons following I/R injury (39). In accordance with that previous study, the present results revealed that the expression level of BCL-2 decreased following $\mathrm{I} / \mathrm{R}$ injury, whereas the expression level of BAX was increased. However, overexpression of COX6B1 significantly reversed the expression levels of BCL-2 and BAX in hippocampal neurons following I/R injury. The present results suggested that COX6B1 overexpression was able to reduce I/R-induced neuronal apoptosis by downregulating the expression level of BAX and by upregulating the expression level of BCL-2. 

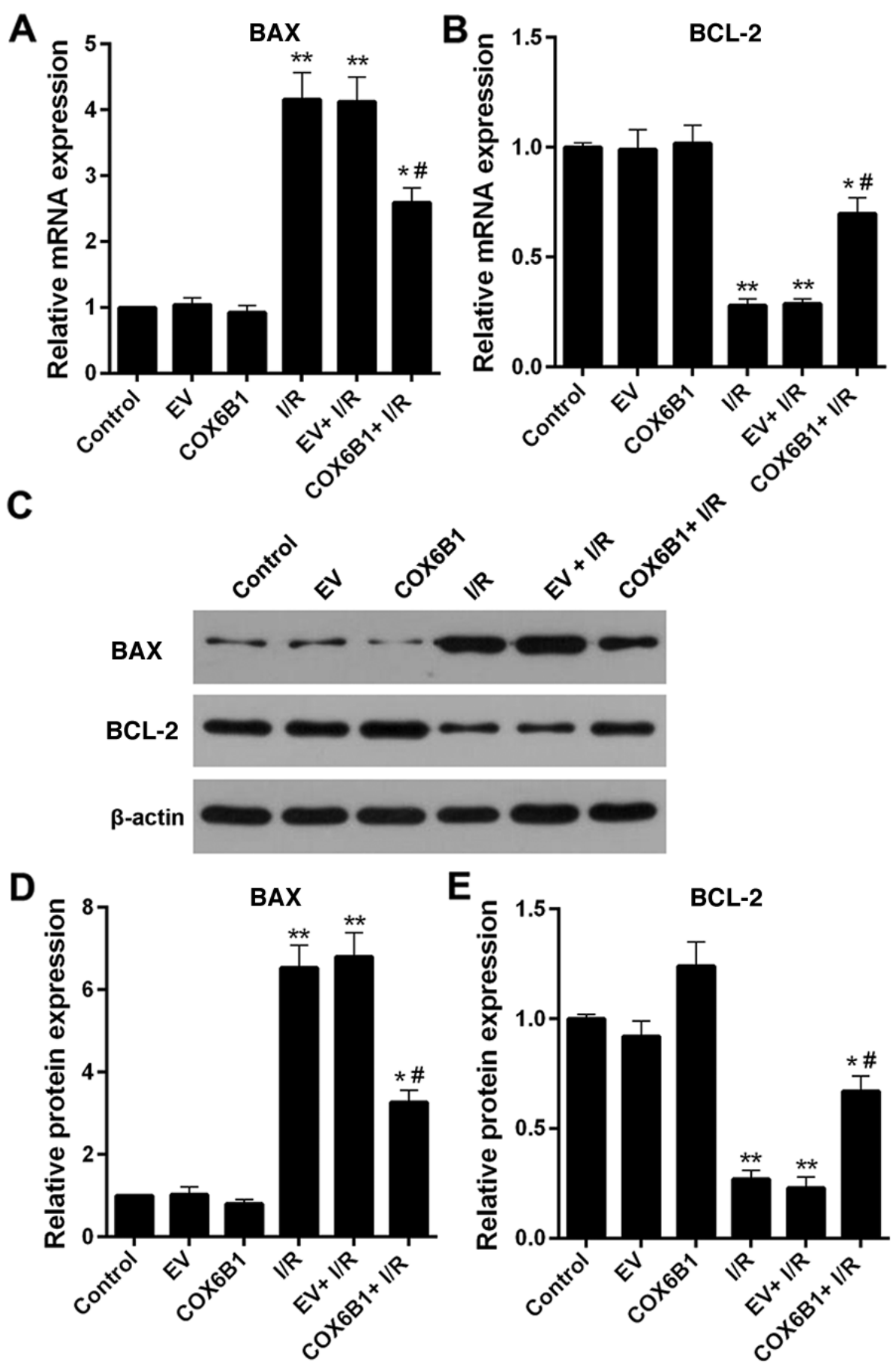

Figure 6. Overexpression of COX6B1 increases the expression levels of BCL-2 and decreases the expression levels of BAX in hippocampal neurons exposed to I/R injury. (A-E) Cells were exposed to OGD for $60 \mathrm{~min}$, reoxygenated for $60 \mathrm{~min}$ and transfected with pcDNA3.1(+)-EV or with pcDNA3.1(+)-COX6B1 overexpression plasmid. (A and B) mRNA expression levels of (A) apoptosis regulator BAX and (B) BCL-2 were determined using reverse transcription-quantitative polymerase chain reaction. (C) Western blotting results of BAX and BCL-2 following I/R injury and COX6B1 overexpression. (D and E) Protein expression levels of (D) BAX and (E) BCL-2 were determined by densitometric analysis. $n=4 ;{ }^{*} \mathrm{P}<0.05$ and ${ }^{* *} \mathrm{P}<0.01$ vs. EV; ${ }^{*} \mathrm{P}<0.05$ vs. EV + I/R. BCL-2, BCL2-associated X, apoptosis regulator; COX6B1, cytochrome c oxidase subunit 6B1; EV, empty vector; I/R, ischemia/reperfusion.

In addition, Wang et al (40) reported that cyt $\mathrm{c}$ was detectable in the cytoplasm of neurons following I/R injury, and appropriate ischemic post-conditioning significantly decreased the cytoplasmic levels of cyt $\mathrm{c}$ in neurons. The release of cyt $\mathrm{c}$ from mitochondria to the cytoplasm is an important step of apoptosis (41-43). Therefore, in the present study, the levels of mitochondrial and cytoplasmic cyt $\mathrm{c}$ in hippocampal neurons were investigated. The results suggested that the expression level of mitochondrial cyt $\mathrm{c}$ decreased; however, the expression level of cytosolic cyt $\mathrm{c}$ increased in hippocampal neurons following I/R injury. This may be a result of the destruction of the mitochondrial outer membrane induced by I/R injury, leading to the release of cyt $\mathrm{c}$ from the mitochondrial membrane space. Conversely, overexpression of COX6B1 reversed the effects of I/R injury; the expression levels of mitochondrial cyt $\mathrm{c}$ in the COX6B1 

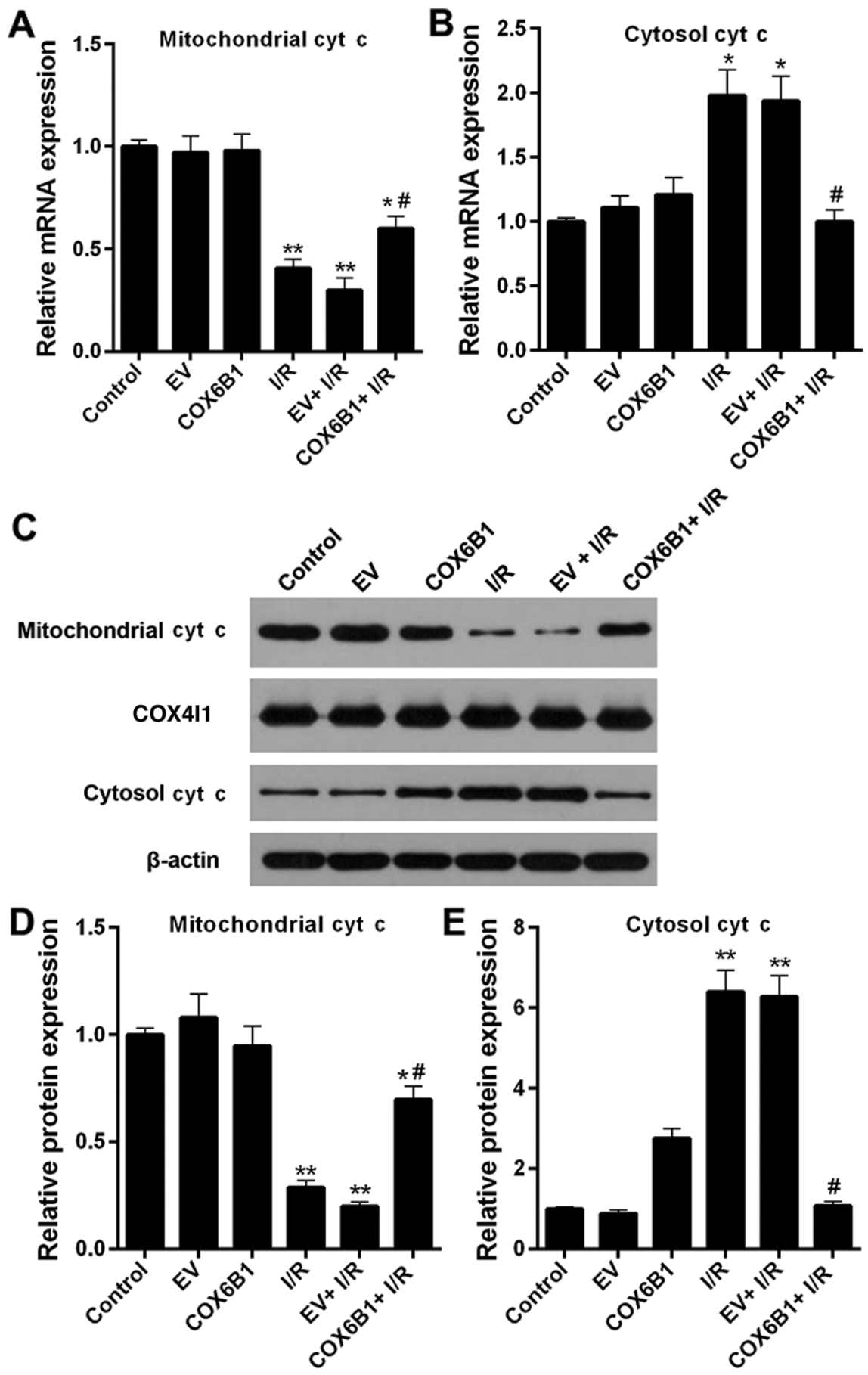

Figure 7. Overexpression of COX6B1 increases mitochondrial cyt c expression and decreases cytosolic cyt c expression in hippocampal neurons following I/R . (A and B) Reverse transcription-quantitative polymerase chain reaction was performed to analyze the mRNA expression levels of cyt $\mathrm{c}$ in (A) mitochondrial and (B) cytosolic fractions of hippocampal neurons. (C-E) Western blotting and densitometric analyses were performed to investigate the protein expression levels of cyt $\mathrm{c}$ and COX4I1 in mitochondrial and cytosolic fractions of hippocampal neurons. $\mathrm{n}=4$; ${ }^{*} \mathrm{P}<0.05$ and ${ }^{* *} \mathrm{P}<0.01 \mathrm{vs}$. EV; ${ }^{*} \mathrm{P}<0.05$ vs. EV $+\mathrm{I} / \mathrm{R}$. COX4I1, cytochrome c oxidase subunit 4I1; COX6B1, cytochrome c oxidase subunit 6B1; cyt c, cytochrome c; EV, empty vector; I/R, ischemia/reperfusion.

$+\mathrm{I} / \mathrm{R}$ group were significantly increased compared with in the $\mathrm{EV}+\mathrm{I} / \mathrm{R}$ group, whereas the expression levels of cytoplasmic cyt $\mathrm{c}$ were significantly decreased in the COX6B1 + $\mathrm{I} / \mathrm{R}$ group compared with in the $\mathrm{EV}+\mathrm{I} / \mathrm{R}$ group. The overexpression of COX6B1 may reduce the IR-induced damage to the mitochondrial outer membrane. Of note, there was no clear difference in the mRNA expression of cytosolic cyt $\mathrm{c}$ between the $\mathrm{EV}$ and the COX6B1 group, whereas the protein expression of cytosolic cyt $\mathrm{c}$ in the COX6B1 group was markedly (but not significantly) increased compared with in the EV group. It may be that the overexpression of COX6B1 promoted a small release of cyt $\mathrm{c}$ from the mitochondria to the cytoplasm; however, this would require further investigation. The present results suggested that COX6B1 overexpression reversed I/R-induced neuronal apoptosis by inhibiting the release of cyt $\mathrm{c}$ from mitochondria to the cytoplasm. However, the present study presents some limitations; the specific molecular mechanism underlying the function 
of COX6B1 in I/R injury was not investigated. Furthermore, the present experiments were performed in vitro and further in vivo analyses are required to confirm the present observations.

Collectively, the present study results indicated that COX6B1 overexpression was able to partially protect neurons following I/R-induced damage by increasing cell viability and by decreasing the levels of cytosolic $\mathrm{Ca}^{2+}$ and apoptosis. The results may facilitate the development of novel strategies for CVD prevention and treatment.

\section{Acknowledgements}

Not applicable.

\section{Funding}

No funding was received.

\section{Availability of data and materials}

The datasets used and/or analyzed during the current study are available from the corresponding author on reasonable request.

\section{Authors' contributions}

SY made substantial contributions to the conception and design of the study. PW, JX and LJ were involved in the acquisition, analysis and interpretation of data. All authors approved of the final version of the manuscript.

\section{Ethics approval and consent to participate}

The animal experiments were approved by The Ethics Committee of The Nanchuan People's Hospital Affiliated to Chongqing Medical University (Chongqing, China).

\section{Patient consent for publication}

Not applicable.

\section{Competing interests}

The authors declare that they have no competing interests.

\section{References}

1. Leira Y, Blanco M, Blanco J and Castillo J: Association between periodontal disease and cerebrovascular disease. A review of the literature. Rev Neurol 61: 29-38, 2015 (In Spanish).

2. Wang Q, Yu H, Jiang C, Sun R, Qi M, Sun S, Xu G, Cai H, Zhang Z, Zhao F, et al: Cerebral infarction as initial presentation in stress cardiomyopathy: Case report and literature review. Medicine (Baltimore) 97: e10804, 2018.

3. Zhang MY, Wu HW, Xu LP and Yang HJ: Pharmacological effect of Schisandrae Chinensis Fructus and relative active components on cardiovascular and cerebrovascular diseases. Zhongguo Zhong Yao Za Zhi 43: 1536-1546, 2018 (In Chinese).

4. Dai L, Song L, Li X, Yang Y, Zheng X, Wu Y, Li C, Zhao H, Wang Y, Wu S and Wang Y: Association of visit-to-visit blood pressure variability with the risk of all-cause mortality and cardiovascular events in general population. J Clin Hypertens (Greenwich) 20: 280-288, 2018.
5. Griffin RL, Falatko SR, Aslibekyan S, Strickland V and Harrigan MR: Aspirin for primary prevention of stroke in traumatic cerebrovascular injury: Association with increased risk of transfusion. J Neurosurg: 1-8, 2018 doi: 10.3171/2017.12. JNS172284. (Epub ahead of print).

6. Endepols H, Mertgens H, Backes H, Himmelreich U, Neumaier B, Graf R and Mies G: Longitudinal assessment of infarct progression, brain metabolism and behavior following anterior cerebral artery occlusion in rats. J Neurosci Methods 253: 279-291, 2015.

7. Struys T, Govaerts K, Oosterlinck W, Casteels C, Bronckaers A Koole M, Van Laere K, Herijgers P, Lambrichts I, Himmelreich U and Dresselaers T: In vivo evidence for long-term vascular remodeling resulting from chronic cerebral hypoperfusion in mice. J Cereb Blood Flow Metab 37: 726-739, 2017.

8. Fan Y,Zhang C, Peng W, Li T, Yin J, Kong Y, Lan C, Li X, Wang R and $\mathrm{Hu} \mathrm{Z}$ : Retraction notice to 'Secretory pathway Ca2+-ATPase isoform 1 knockdown promotes Golgi apparatus stress injury in a mouse model of focal cerebral ischemia-reperfusion: In vivo and in vitro study' [Brain Res. 1642 (2016) 189-196]. Brain Res 1670: 253, 2017.

9. Yang ML, Tao T, Xu J, Liu Z and Xu D: Antiapoptotic effect of gene therapy with recombinant adenovirus vector containing hypoxia-inducible factor- $1 \alpha$ after cerebral ischemia and reperfusion in rats. Chin Med J (Engl) 130: 1700-1706, 2017.

10. Hossmann KA: The two pathophysiologies of focal brain ischemia: Implications for translational stroke research. J Cereb Blood Flow Metab 32: 1310-1316, 2012.

11. Mui K, Yoo AJ, Verduzco L, Copen WA, Hirsch JA, González RG and Schaefer PW: Cerebral blood flow thresholds for tissue infarction in patients with acute ischemic stroke treated with intra-arterial revascularization therapy depend on timing of reperfusion. AJNR Am J Neuroradiol 32: 846-851, 2011.

12. Lenka N, Vijayasarathy C, Mullick J and Avadhani NG: Structural organization and transcription regulation of nuclear genes encoding the mammalian cytochrome c oxidase complex. Prog Nucleic Acid Res Mol Biol 61: 309-344, 1998.

13. Barrientos A, Barros MH, Valnot I, Rötig A, Rustin P and Tzagoloff A: Cytochrome oxidase in health and disease. Gene 286: 53-63, 2002.

14. Huttemann M, Jaradat S and Grossman LI: Cytochrome c oxidase of mammals contains a testes-specific isoform of subunit VIb-the counterpart to testes-specific cytochrome c? Mol Reprod Dev 66: 8-16, 2003.

15. Lu J, Wang K, Rodova M, Esteves R, Berry D, E L, Crafter A, Barrett M, Cardoso SM, Onyango I, et al: Polymorphic variation in cytochrome oxidase subunit genes. J Alzheimers Dis 21: 141-154, 2010.

16. Massa V, Fernandez-Vizarra E, Alshahwan S, Bakhsh E, Goffrini P, Ferrero I, Mereghetti P, D'Adamo P, Gasparini P and Zeviani M: Severe infantile encephalomyopathy caused by a mutation in COX6B1, a nucleus-encoded subunit of cytochrome c oxidase. Am J Hum Genet 82: 1281-1289, 2008.

17. Abdulhag UN, Soiferman D, Schueler-Furman O, Miller C, Shaag A, Elpeleg O, Edvardson S and Saada A: Mitochondrial complex IV deficiency, caused by mutated COX6B1, is associated with encephalomyopathy, hydrocephalus and cardiomyopathy. Eur J Hum Genet 23: 159-164, 2015.

18. Kim SE, Mori R, Komatsu T, Chiba T, Hayashi H, Park S, Sugawa MD, Dencher NA and Shimokawa I: Upregulation of cytochrome c oxidase subunit 6b1 (Cox6b1) and formation of mitochondrial supercomplexes: Implication of Cox6b1 in the effect of calorie restriction. Age (Dordr) 37: 9787, 2015.

19. Popovic DM: Current advances in research of cytochrome c oxidase. Amino Acids 45: 1073-1087, 2013.

20. Feng Y, Madungwe NB, da Cruz Junho CV and Bopassa JC: Activation of $G$ protein-coupled oestrogen receptor 1 at the onset of reperfusion protects the myocardium against ischemia/reperfusion injury by reducing mitochondrial dysfunction and mitophagy. Br J Pharmacol 174: 4329-4344, 2017.

21. Zhang W, Wang Y, Wan J, Zhang P and Pei F: COX6B1 relieves hypoxia/reoxygenation injury of neonatal rat cardiomyocytes by regulating mitochondrial function. Biotechnol Lett 41: 59-68, 2019.

22. Livak KJ and Schmittgen TD: Analysis of relative gene expression data using real-time quantitative PCR and the 2(-Delta Delta C(T)) method. Methods 25: 402-408, 2001.

23. Facci L and Skaper SD: Culture of rodent cortical, hippocampal, and striatal neurons. Methods Mol Biol 1727: 39-47, 2018. 
24. Rivera-Carvantes MC, Jarero-Basulto JJ, Feria-Velasco AI, Beas-Zarate C, Navarro-Meza M, Gonzalez-Lopez MB, Gudino-Cabrera G and Garcia-Rodriguez JC: Changes in the expression level of MAPK pathway components induced by monosodium glutamate-administration produce neuronal death in the hippocampus from neonatal rats. Neuroscience 365: 57-69, 2017.

25. Caceres A, Banker G, Steward O, Binder L and Payne M: MAP2 is localized to the dendrites of hippocampal neurons which develop in culture. Brain Res 315: 314-318, 1984.

26. Lei X, Lei L, Zhang Z and Cheng Y: Diazoxide inhibits of ER stressmediated apoptosis during oxygenglucose deprivation in vitro and cerebral ischemiareperfusion in vivo. Mol Med Rep 17: 8039-8046, 2018.

27. Lin YW, Chen TY, Hung CY, Tai SH, Huang SY, Chang CC, Hung HY and Lee EJ: Melatonin protects brain against ischemia/reperfusion injury by attenuating endoplasmic reticulum stress. Int J Mol Med 42: 182-192, 2018.

28. Baden KN, Murray J, Capaldi RA and Guillemin K: Early developmental pathology due to cytochrome c oxidase deficiency is revealed by a new zebrafish model. J Biol Chem 282: 34839-34849, 2007.

29. Liu W, Gnanasambandam R, Benjamin J, Kaur G, Getman PB, Siegel AJ, Shortridge RD and Singh S: Mutations in cytochrome c oxidase subunit VIa cause neurodegeneration and motor dysfunction in Drosophila. Genetics 176: 937-946, 2007.

30. Su F, Guo AC, Li WW, Zhao YL, Qu ZY, Wang YJ, Wang Q and Zhu YL: Low-dose ethanol preconditioning protects agains oxygen-glucose deprivation/reoxygenation-induced neuronal injury by activating large conductance, $\mathrm{Ca}^{2+}$-Activated $\mathrm{K}^{+}$channels in vitro. Neurosci Bull 33: 28-40, 2017.

31. Wang Y, Shen Y, Lin HP, Li Z, Chen YY and Wang S: Large-conductance $\mathrm{Ca}(2+)$-activated $\mathrm{K}(+)$ channel involvement in suppression of cerebral ischemia/reperfusion injury after electroacupuncture at Shuigou (GV26) acupoint in rats. Neural Regen Res 11: 957-962, 2016.

32. Savigni DL, O'Hare Doig RL, Szymanski CR, Bartlett CA, Lozic I, Smith NM and Fitzgerald M: Three $\mathrm{Ca} 2+$ channel inhibitors in combination limit chronic secondary degeneration following neurotrauma. Neuropharmacology 75: 380-390, 2013.

33. Zolezzi JM, Carvajal FJ, Rios JA, Ordenes D, Silva-Alvarez C, Godoy JA and Inestrosa NC: Tetrahydrohyperforin induces mitochondrial dynamics and prevents mitochondrial $\mathrm{Ca} 2+$ overload after $\mathrm{A} \beta$ and $\mathrm{A} \beta$-AChE complex challenge in rat hippocampal neurons. J Alzheimers Dis 37: 735-746, 2013.

34. Ma YY, Li KY, Wang JJ, Huang YL, Huang Y and Sun FY: Vascular endothelial growth factor acutely reduces calcium influx via inhibition of the $\mathrm{Ca} 2+$ channels in rat hippocampal neurons. J Neurosci Res 87: 393-402, 2009.
35. Li J, Yan D, Liu X, Wang Y, Zhao X, Zhang Y and Zhang C: U0126 protects hippocampal CA1 neurons against forebrain ischemia-induced apoptosis via the ERK1/2 signaling pathway and NMDA receptors. Neurol Res 40: 318-323, 2018.

36. YuZ,Cai M,LiX,ZhangJ,WuT, Yang F,ZhuW, Xiang Y,Zhang W, Xiang J and Cai D: Neuroprotective effects of Tongxinluo on focal cerebral ischemia and reperfusion injury in rats associated with the activation of the MEK1/2/ERK1/2/p90RSK signaling pathway. Brain Res 1685: 9-18, 2018

37. Martinou JC and Youle RJ: Mitochondria in apoptosis: Bcl-2 family members and mitochondrial dynamics. Dev Cell 21: 92-101, 2011.

38. Ouyang YB and Giffard RG: MicroRNAs affect BCL-2 family proteins in the setting of cerebral ischemia. Neurochem Int 77: $2-8,2014$.

39. Aboutaleb N, Shamsaei N, Rajabi H, Khaksari M, Erfani S, Nikbakht F, Motamedi P and Shahbazi A: Protection of hippocampal CA1 neurons against ischemia/reperfusion injury by exercise preconditioning via modulation of $\mathrm{Bax} / \mathrm{Bcl}-2$ ratio and prevention of caspase-3 activation. Basic Clin Neurosci 7: 21-29, 2016.

40. Wang JY, Shen J, Gao Q, Ye ZG, Yang SY, Liang HW, Bruce IC, Luo BY and Xia Q: Ischemic postconditioning protects against global cerebral ischemia/reperfusion-induced injury in rats. Stroke 39: 983-990, 2008.

41. Antonawich FJ: Translocation of cytochrome c following transient global ischemia in the gerbil. Neurosci Lett 274: 123-126, 1999.

42. Muranyi M and Li PA: Bongkrekic acid ameliorates ischemic neuronal death in the cortex by preventing cytochrome $\mathrm{c}$ release and inhibiting astrocyte activation. Neurosci Lett 384: 277-281, 2005.

43. Zhao H, Yenari MA, Cheng D, Sapolsky RM and Steinberg GK: Biphasic cytochrome c release after transient global ischemia and its inhibition by hypothermia. J Cereb Blood Flow Metab 25: 1119-1129, 2005

This work is licensed under a Creative Commons Attribution-NonCommercial-NoDerivatives 4.0 International (CC BY-NC-ND 4.0) License. 